\title{
Evaluation of the central corneal thickness, retinal thickness and intraocular pressure in patients with euthyroid Hashimoto's thyroiditis
}

\author{
Fatma Neslihan Cuhacı Seyrek ${ }^{1}$, Hüsniye Başer ${ }^{1}$, Nagihan Uğurlu ${ }^{2}$, Fatma Yülek ${ }^{2}$, Reyhan Ersoy ${ }^{1}$, \\ Nurullah Çağıl ${ }^{2}$, Bekir Çakır ${ }^{1}$ \\ (1) Ankara Yildirim Beyazit University, Faculty of Medicine, Endocrinology and Metabolism, Ankara, Turkey \\ (2) Ankara Yildirim Beyazit University, Faculty of Medicine, Ophthalmology, Ankara, Turkey
}

Date submitted:

Oct 18, 2018

Date accepted:

Dec 03, 2018

Online publication date:

June 15, 2019

\section{Corresponding Author: \\ Fatma Neslihan Cuhaci Seyrek \\ Ankara Yıldırım Beyazıt \\ University, Faculty of Medicine, \\ Endocrinology and Metabolism, \\ Ankara, Turkey \\ neslihan_cuhaci@yahoo.com}

Keywords: Euthyroid Hashimoto's thyroiditis, central corneal thickness, retinal thickness, intraocular pressure.

\begin{abstract}
Aims:There are few data concerning the effects of thyroid disorders on intraocular pressure (IOP), central corneal thickness (CCT) and retinal thickness (RT) and to our knowledge no data have been reported in Hashimoto's thyroiditis (HT). In this study we aimed to evaluate the alterations in IOP, CCT and RT in patients with euthyroid $\mathrm{HT}$.
\end{abstract}

Methods:In this cross-sectional study we examined IOP, CCT and RT in patients with euthyroid $\mathrm{HT}$ and age and gender matched controls.

Results:We evaluated 80 eyes of 40 patients with euthyroid HT and 96 eyes of 48 controls. Serum sensitive thyroid stimulating hormone (sTSH) $(2.10 \pm 1.04$ vs $1.92 \pm 1.23 \mathrm{ulU} / \mathrm{ml})$, free triiodothyronine (fT3) $(3.19 \pm 0.43$ vs $3.14 \pm 0.34 \mathrm{pg} / \mathrm{ml})$, and free tetraiodothyronine (fT4) $(1.20 \pm 0.14$ vs $1.18 \pm 0.13 \mathrm{ng} / \mathrm{dl})$ levels were similar between the two groups. No statistically significant difference was observed in mean RT (266.93 \pm 26.46 vs $275.35 \pm 38.81 \mu \mathrm{m})$, CCT $(533.68 \pm 29.28$ vs $540.06 \pm 29.13 \mu \mathrm{m})$, or IOP $(13.98 \pm 2.42$ vs $14.32 \pm 2.62 \mathrm{mmHg})$ between HT patients and controls. In the HT group Anti-TPO was negatively correlated with mean RT ( $r=$ $-0.227, p=0.033)$.

Conclusions:We have found no differences in CCT, RT and IOP between euthyroid HT patients and controls. In the present study, however there was a negative correlation between the antiTPO levels and RT.

\section{Introduction}

Hashimoto's thyroiditis (HT), which was first described over a century ago is defined as a chronic inflammatory condition of the thyroid gland. It is the most common cause of hypothyroidism and considered the most common autoimmune disease and a common endocrine disorder (1-3). Combination of clinical features, the presence of serum antibodies against thyroid antigens such as thyroid peroxidase (TPO) and thyroglobulin (TG), and ultrasonography findings are the identifiers of the HT (4). The sonographic features of HT include; a diffusely enlarged thyroid gland, coarsened parenchymal echogenicity, more hypoechoic images than normal, often hypervascular on color Doppler ultrasonography, as well as the presence of a micronodular pattern, which is a strong indicator (5-6).

The best serological marker to identify the HT is thought to be the circulating antibodies against TPO (anti-TPO), which are found in about $95 \%$ of HT patients but rarely in healthy controls (4). Antibodies to TG (anti-TG) are less sensitive (positive in only $60-80 \%$ of HT patients) and less specific than TPO antibodies (4). There are some groups of patients who are in a euthyroid state but have elevated thyroid antibody levels (7).

The eye is one of the organ which have predisposition of the impacts of various systemic disorders (8) such as hypothyroidism and hyperthyroidism. Thyroid hormones take an essential role in the neural development of the eye, particularly for normal development of the retina and the success of color vision (8). It also organizes the intrinsic mechanisms to handle the retinal cytoarchitecture and layering (9). It has been reported that acquired deficiency of thyroid hormones affects the intraocular pressure (IOP), as well as orbitopathies, such as periorbital edema and chemosis associated to myxedema (10). Centanni et al. (11) showed a reversible increase in IOP even in subclinical hypothyroidism; this finding brought about the question of whether some microscopic findings precede the macroscopic findings of hypothyroidism. Increased IOP and visual field defects can actually be seen in cases of severe Graves ophthalmopathy (12). The effects of thyroid disorders on the central corneal thickness (CCT) are not well defined in the literature. Central corneal thickness has been demonstrated to play a role in the interpretation of IOP (12). 
There are few data regarding the effects of hypo- and hyperthyroidism on CCT and IOP, and to our knowledge, there is no reported data in patients with euthyroid HT. The aim of our study was to evaluate the alterations in IOP, CCT and retinal thickness (RT) in patients with euthyroid HT as to whether or not it is necessary to assess the need for follow-up in these patients from an ophthalmological point of view.

\section{Methods}

\section{Patients}

This cross-sectional study was conducted in 80 eyes of 40 patients with euthyroid HT and 96 eyes of 48 healthy subjects as controls. The diagnosis of HT was based on positive anti -TPO and anti-TG antibodies and sonographic features of the thyroid. The patients included were all euthyroid in both clinical and laboratory tests (free triiodothyronine (fT3), free tetraiodothyronine (fT4) levels and sensitive thyroid stimulating hormone (sTSH) levels were within the normal range) and had positive anti-TPO and anti-TG antibodies and moderate-to-severe parenchymal hypoechogenicity $(7,13)$, and a micronodular pattern (6) by thyroid ultrasound. The control group consisted of healthy individuals with no history of thyroid disease.

Exclusion criteria were patients with systemic diseases (e.g., diabetes mellitus, hypertension, chronic renal failure, or chronic hepatic failure), patients with chronic ocular diseases (e.g., refraction defects, glaucoma, uveitis, keratitis, dry eye syndrome, or conjunctivitis), those using contact lenses, those with previous optic nerve damage, and those who had undergone laser treatment or systemic or topical corticosteroid use, immunosuppressive therapy, intravitreal injections to the eyes, ocular surgery, or trauma. Patients under 18 years old and those unwilling to participate were also excluded.

The study was approved by the local ethics board. Informed consent was obtained from each participant. The study protocol followed the tenets of the 1964 Declaration of Helsinki.

\section{Laboratory tests}

Levels of sTSH, fT3, fT4, and thyroid autoantibodies (anti-TPO and anti-TG) were measured in all patients using chemiluminescence methods (Immulite 2000; Diagnostic Products Corporation, Los Angeles, CA and UniCel DxI 800; Beckman Coulter, Brea, CA). The normal ranges for sTSH, fT3, fT4, anti-TPO, and anti-TG were 0.27-4.2 $\mathrm{\mu lU} / \mathrm{mL}, 1.8-4.6 \mathrm{pg} / \mathrm{mL}$, 0.9-1.7 ng/dL, 0-34 IU/ml, and 0-115 IU/ml, respectively.
An Esaote color Doppler ultrasound (US) system (Model 796FDII; MAG Technology Co. Ltd., Yung-Ho City, Taipei, Taiwan) and a standard US system with a superficial probe (Model LA523 13-4, 5.5 and $12.5 \mathrm{MHz}$ ) were used. Chronic thyroiditis was defined when the thyroid gland was diffusely heterogeneous and/or hypoechoic with/without pseudonodules and fibrotic bands.

\section{Ocular evaluation}

A single specialist who is blinded to patient status was performed the ophthalmological examination, which includes CCT, $\mathrm{RT}$, and IOP. Retinal thickness in the central circle (diameter, 1 $\mathrm{mm}$ ) was calculated after pupil dilatation using Spectralis SDOCT (Heidelberg Engineering, Heidelberg, Germany). Central corneal thickness was measured by non-contact pachymetry (Nidek NT 530P, Nidek Co., Ltd., Aichi, Japan). The mean value of three consecutive measurements from the central cornea was used for CCT assessment. Intraocular pressure was measured using Goldmann applanation tonometry.

\section{Statistical analysis}

All statistical analyses were performed with the SPSS software (ver. 15.0; SPSS Inc., Chicago, IL, USA). Descriptive analyses are presented using means \pm standard deviation (SD) for normally distributed variables, and medians and range (min-max) for non-normally distributed variables. The $x^{2}$ test was used to investigate differences between groups regarding categorical variables. Comparisons between groups were performed using Student's t-test for parametric variables and the Mann-Whitney U-test for non-parametric variables. Pearson's and Spearman's correlation analyses were performed to assess possible associations between parametric and non-parametric variables, respectively. A p-value $<0.05$ was considered to indicate statistical significance.

\section{Results}

In total, 40 patients (2 males, 38 females) with euthyroid HT, with a mean age of $41.95 \pm 10.47$ years and 48 (4 males, 44 females) healthy subjects, with a mean age of $43.50 \pm 9.99$ years, were evaluated. There was no significant difference between patients and control group with regard to age or gender (all $p>0.05$ ). Serum sTSH, fT3, and fT4 levels were similar between the two groups. Anti-TPO and anti-TG levels of euthyroid HT patients were significantly higher than those of the controls (each $p<0.001$ ). Demographical and biochemical data of the study group are given in Table 1. No statistically significant dif-

Table 1. Demographical, biochemical, and ocular data of the patients and control groups

\begin{tabular}{lccc}
\hline Variables & Patients $(\mathbf{n = 4 0})$ & Controls $(\mathbf{n = 4 8 )}$ & p-value \\
\hline Age $($ years $)$ & $41.95 \pm 10.47$ & $43.50 \pm 9.99$ & 0.480 \\
\hline Female/Male & $38 / 2$ & $44 / 4$ & 0.537 \\
\hline fT3 $(\mathrm{pg} / \mathrm{ml})$ & $3.19 \pm 0.43$ & $3.14 \pm 0.34$ & 0.567 \\
\hline fT4 $(\mathrm{ng} / \mathrm{dl})$ & $1.20 \pm 0.14$ & $1.18 \pm 0.13$ & 0.416 \\
\hline sTSH $(\mathrm{ulU} / \mathrm{ml})$ & $2.10 \pm 1.04$ & $1.92 \pm 1.23$ & 0.475 \\
\hline Anti-TPO $(\mathrm{IU} / \mathrm{ml})$ & $97.94(35-1000)$ & $8.15(5-32.3)$ & $<0.001$ \\
\hline Anti-TG $(\mathrm{IU} / \mathrm{ml})$ & $178.30(118.6-4000)$ & $17.98(10-100.40)$ & $<0.001$ \\
\hline Mean RT $(\mu \mathrm{m})$ & $266.93 \pm 26.46$ & $275.35 \pm 38.81$ & 0.101 \\
\hline Mean CCT $(\mu \mathrm{m})$ & $533.68 \pm 29.28$ & $540.06 \pm 29.13$ & 0.161 \\
\hline Mean IOP $(\mathrm{mmHg})$ & $13.98 \pm 2.42$ & $14.32 \pm 2.62$ & 0.390 \\
\hline $\begin{array}{l}\text { fT3: free triiodothyronine, fT4: free tetraiodothyronine, sTSH: sensitive thyroid stimulating hormone, Anti-TPO: anti-thyroid peroxidase, Anti-TG: anti-thyroglobulin RT: retinal thickness, CCT: } \\
\text { central corneal thickness, IOP: intraocular pressure. }\end{array}$ & & & \\
\hline
\end{tabular}




\begin{tabular}{|c|c|c|c|}
\hline Variables & $\begin{array}{c}\text { sTSH }<2.5 \text { (group A) } \\
(n=62)\end{array}$ & $\begin{array}{c}\text { sTSH } \geq 2.5 \text { (group B) } \\
(n=26)\end{array}$ & p-value \\
\hline $\mathrm{RT}(\mu \mathrm{m})$ & $269.33 \pm 36.18$ & $276.76 \pm 27.47$ & 0.185 \\
\hline $\mathrm{CCT}(\mu \mathrm{m})$ & $536.36 \pm 28.41$ & $538.54 \pm 31.60$ & 0.666 \\
\hline IOP (mmHg) & $14.17 \pm 2.51$ & $14.12 \pm 2.57$ & 0.909 \\
\hline
\end{tabular}

Table 3. Correlations between thyroid function tests and retinal thickness, central corneal thickness and intraocular pressure

\begin{tabular}{|c|c|c|c|c|c|c|c|c|c|c|}
\hline & \multicolumn{2}{|c|}{ sTSH } & \multicolumn{2}{|c|}{ fT3 } & \multicolumn{2}{|c|}{$\mathrm{ft} 4$} & \multicolumn{2}{|c|}{ Anti- TPO } & \multicolumn{2}{|l|}{ Anti-TG } \\
\hline & $\mathbf{r}$ & $p$ & $\mathbf{r}$ & $p$ & $\mathbf{r}$ & p & $\mathbf{r}$ & p & $\mathbf{r}$ & $\mathbf{p}$ \\
\hline Mean RT & 0.118 & 0.274 & 0.054 & 0.616 & 0.223 & 0.370 & -0.227 & 0.033 & 0.004 & 0.970 \\
\hline Mean CCT & -0.085 & 0.445 & -0.079 & 0.478 & 0.059 & 0.595 & -0.028 & 0.801 & -0.136 & 0.221 \\
\hline Mean IOP & -0.015 & 0.894 & 0.054 & 0.628 & -0.096 & 0.395 & 0.017 & 0.877 & -0.010 & 0.925 \\
\hline
\end{tabular}

ference was observed in mean RT, CCT, or IOP between the groups (all $p>0.05$; Table 1).

The patient group was re-evaluated according the STSH level. When we used a sTSH cut-off of 2.5 , specifically $\mathrm{STSH}<2.5$ (group $\mathrm{A}$ ) versus $\mathrm{TSH} \geq 2.5$ (group $\mathrm{B}$ ), no significant difference was found between the groups in mean RT, CCT, or IOP (all $p$ > 0.05; Table 2).

Anti-TPO was negatively correlated with mean RT $(r=$ $-0.227, p=0.033$ ). However, there was no significant correlation between anti-TPO levels and CCT or IOP (Table 3). Also, no significant correlation was found between anti-TG levels, and mean RT, CCT, or IOP (all p > 0.05).

\section{Discussion}

There have been conflicting results related to the effects of thyroid hormone on CCT and IOP (14). The first reports related with the IOP rise in hypothyroidism were released for publication in 1897 and has been associated to hypothalamic disturbances, either directly or by the mediation of the pituitary gland acting on the thyroid and eye at the same time. Glaucoma has been related at times with thyrotoxicosis and at times with myxedema (15). Cheng and Perkins suggested that there may be a genetic predisposition to both conditions (15). In 1965, McLenachan and Davies proposed that the deposition of glycosaminoglycans in the trabecular meshwork may result with a decrease in aqueous humor outflow (16). Later, Becker et al. (17) brought out the question as to whether or not hypothyroidism induced myxedema of the trabecular meshwork. Smith et al. (18) suggested that vasculopathy can change the ocular blood flow as the mechanism of the IOP rise in hypothyroidism. Glycosaminoglycan deposition in the trabecular meshwork seems to be the most noteworthy (8).

The diagnosis of glaucoma is carried out by a fundus examination and visual field testing, typically in the presence of elevated IOP $(15,16)$. It is the second leading cause of blindness in the worldwide and usually assessed with Goldmann applanation tonometry (GAT), which is the gold standard for IOP measurements $(19,20)$. Several reports indicate the higher prevalence of primary open angle glaucoma (POAG) among hypothyroid patients; however, in contrast, other reports have failed to support this $(18,21,22,23)$. Smith et al. (18) detemined an IOP decrease with treatment of hypothyroidism in a patient with POAG, and they associated the IOP rise to a reduction in outflow in hypothyroid patients (24-25). In the study of Ozturk et al.(8), they found no significant IOP decrease with treatment in hypothyroid patients and also no correlation between IOP measurements and the decrease in TSH. Bahceci et al. (26) demonstrated a significant decrease in IOP with treatment; however, it was not correlated with changes in thyroid hormone levels.

In patients with thyroid eye disease, Rahman et al.(27) found an average difference of $1 \mathrm{mmHg}$ between the IOP measured with GAT and the TonoPen. The authors suggested that there may be various factors affecting this small difference in IOP reading, such as limbal concavity, wall stiffness, and peripheral corneal thickness (27). In this study we used the GAT. GAT measurements can be influenced by several ocular properties, such as CCT, axial length, and corneal curvature as shown in several studies (28-29). In our study, we found no correlation in IOP between euthyroid HT patients and the control subjects, also no differences were found according the sTSH levels. Such possible factors and euthyroid state may be responsible for the unsignificant results in our study. Reagarding with the Goldmann equation, IOP is positively correlated with aqueous inflow and episcleral venous pressure, and negatively correlated with outflow facility (30). In Graves' orbitopathy elevated episcleral venous pressure levels were shown, and raised retrobulbar pressure, above normal venous pressure, has been reported as a possible cause of reduced orbital venous drainage, which may increase the IOP (30).

Central corneal thickness is closely related with IOP (19). The normal CCT value in the human cornea is accepted as 536 $\pm 0.31 \mu \mathrm{m}$ in the literature (29). In previous studies, IOP measurements were corrected using the formula given by Doughty (29). In our study, no such correction was necessary, because the mean CCTs were similar in both groups. In Bahceci et al. study (26) they have showed an increased IOP and CCT in 
hypothyroid patients and a decrease with replacement therapy. Since the corneal thickness decreased following replacement therapy they proposed that these reversible changes (both IOP and CCT decreased with treatment) may be associated to mucopolysaccharide deposition in the corneal stroma (26). In contrast, Ozturk et al.(8) found no change in IOP or CCT with treatment in hypothyroid patients. Ozturk et al. (8) suggested that these conflicting results may be due to the different measurement methods: Bahceci et al. (26) used ultrasonic pachymetry while Ozturk et al. used a Scheimpflug camera (8). Ozturk et al. found no significant correlation between the change in TSH level and the change in CCT after the 6 months of treatment (8). Additionally, there was no significant change in the mean RT after replacement therapy, and the correlation of RT with the decrease of TSH was also not significant (8). Similarly, Atmaca et al. (14), showed no correlation between thyroid hormone and CCT or IOP. In the study of Konuk et al. (12) they evaluated CCT alterations in Graves' disease and GO cases regarding with disease severity and hormonal status of the patients and control participants. They revealed that the CCT values of patients with Graves' disease and patients with GO with hyperthyroid and euthyroid hormonal status showed no statistical difference among themselves or versus control participants. Also, no significant difference was found in the IOP values of the patients (12). Finally, their study revealed that neither hyperthyroidism nor severity of orbital disease affected CCT (12). Conflicting results may be attributed to an unknown period of a hypothyroid state, a younger mean age, and the lack of orbitopathy (8). Furthermore, speculations are carried out related with individuals who have prone to autoimmunity also being susceptible to thyroid disorders and glaucoma (8). In our study we found no significant differences in CCT and RT between euthyroid HT patients and control subjects and also according the STSH levels.

The only significant result of our study was a negative correlation between anti-TPO levels and mean RT. But no correlation was found between anti-Tg levels and retinal thickness. Therefore, it is is not possible to mention a causal relationship between autoimmunity and retinal thickness. Further studies will have to be required to confirm that relation and explanation.

CONCLUSIONS: To our knowledge this is the first study to evaluate the relationship between euthyroid $\mathrm{HT}$ patients and $\mathrm{RT}$, CCT, and IOP, and we have found no significant difference in the CCT, RT, or IOP between HT patients and control subjects. This outcome may have been due to the euthyroid state of the patients, individual differences in the patients (e.g., limbal concavity, wall stiffness, or peripheral corneal thickness), and genetic susceptibility, as mentioned above. But our study revealed a negative correlation between anti-TPO levels and RT. Therefore, we can not exactly say that it is not necessary to follow-up the euthyroid HT patients in an ophthalmological view, in contrast it may be usefull in these group of patients especially concerning with RT. To clarify this, future studies with larger sample sizes are required.

Acknowledgements: Author contributions: Concept: N.S and H.B, design: N.S, patient selection: N.S and H.B, ophthalmologic evaluation: N.U and F.Y, analysis: N.S and H.B, literature search, writing: N.S and H.B, critical review: R.E, N.C and B.C. No financial support was received from any source for this work.

Conflict of Interest: The authors declared they do not have anything to disclose regarding conflict of interest with respect to this manuscript.

\section{References}

1. McLeod DS, Cooper DS. The incidence and prevalence of thyroid autoimmunity. Endocrine. 2012; 42(2):252265.

2. Ceylan I, Yener S, Bayraktar F, Secil M. Roles of ultrasound and power Doppler ultrasound for diagnosis of Hashimoto thyroiditis in anti-thyroid marker-positive euthyroid subjects. Quant Imaging Med Surg. 2014; 4(4):232-238.

3. Bircan HY, Koc B, Akarsu C, et al. Is Hashimoto's thyroiditis a prognostic factor for thyroid papillary microcarcinoma? Eur Rev Med Pharmacol Sci. 2014; 18 (13):1910-1915.

4. Caturegli P, De Remigis A, Rose NR. Hashimoto thyroiditis: clinical and diagnostic criteria. Autoimmun Rev. 2014; 13(4-5):391-397.

5. Pedersen OM, Aardal NP, Larssen TB, Varhaug JE, Myking O,Vik-Mo $\mathrm{H}$. The value of ultrasonography in predicting autoimmune thyroid disease. Thyroid. 2000; 10(3):251-259.

6. Yeh HC, Futterweit W, Gilbert P. Micronodulation: ultrasonographic sign of Hashimoto thyroiditis. J Ultrasound Med. 1996; 15(12):813-819.

7. Acar T, Ozbek SS, Erdogan M, Ozgen AG, Demirel SO. US findings in euthyroid patients with positive antithyroid autoantibody tests compared to normal and hypothyroid cases. Diagn Interv Radiol. 2013; 19(4):265-270.

8. Ozturk BT, Kerimoglu H, Dikbas O, Pekel H, Gonen MS. Ocular changes in primary hypothyroidism. BMC Res Note. 2009; 29;2:266.

9. Pinazo-Durán MD, Iborra FJ, Pons S, Sevilla-Romero E, Gallego-Pinazo R, Muñoz A. Postnatal thyroid hormone supplementation rescues developmental abnormalities induced by congenital-neonatal hypothyroidism in the rat retina. Ophthalmic Res. 2005; 37:225-234.

10. Wartofsky L. Diseases of the thyroid. In Fauci SA, Braunwald E (eds) Harrison's Principles of Internal Medicine, 14th edn. The McGraw Hill Companies, Philadelphia, pp 212-2035. 1998.

11. Centanni M, Cesareo R, Verallo O, et al. Reversible increase of intraocular pressure in subclinical hypothyroid patients. Eur J Endocrinol. 1997;136(6):595-598.

12. Konuk O, Aktas Z, Aksoy S, Onol M, Unal M. Hyperthyroidism and severity of orbital disease do not change the central corneal thickness in Graves' ophthalmopathy. Eur J Ophthalmol. 2008; 18(1):125-127.

13. Marcocci C, Vitti P, Cetani F, Catalano F, Concetti R, Pinchera A. Thyroid ultrasonography helps to identify patients with diffuse lymphocytic thyroiditis who are prone to develop hypothyroidism. J Clin Endocrinol Metab. 1991; 72(1):209-213.

14. Atmaca M, Kızıldağ E, Candan Z, Özbay MF, Seven I. Ocular findings in Sheehan's syndrome. Graefes Arch Clin Exp Ophthalmo. 2015; 253(5):759-763. 
15. Cheng $\mathrm{H}$, Perkins ES. Thyroid disease and glaucoma. $\mathrm{Br}$ J Ophthalmol. 1967; 51(8):547-553.

16. McLenechan J, Davies DM. Glaucoma nad the thyroid. Br J Ophthalmol, 1965; 49(8): 441-448.

17. Becker B, Holker AE, Ballin N. Thyroid function and glaucoma. Am J Ophthalmol, 1966; 61(5): 997-999.

18. Smith KD, Arthurs BP, Saheb N. An association between hypothyroidism and primary open-angle glaucoma. Ophthalmology. 1993; 100(10):1580-1584.

19. Polat SB, Ugurlu N, Ersoy R, Oguz O, Duru N, Cakır $B$. Evaluation of central corneal and central retinal thicknesses and intraocular pressure in acromegaly patients. Pituitary. 2014; 17(4):327-332.

20. Baser H, Cuhaci N, Topaloglu O, et al. Is there any association between primary hyperparathyroidism and ocular changes, such as central corneal thickness, retinal thickness, and intraocular pressure ? Endocrine. 2016;51(3):545-550.

21. Muñoz-Negrete FJ, Rebolleda G, Almodóvar F, Díaz B, Varela C. Hypothyroidism and primary open-angle glaucoma. Ophthalmologica. 2000; 214(5):347-349.

22. Girkin CA, McGwin G Jr, McNeal SF, Lee PP, Owsley C. Hypothyroidism and the development of open-angle glaucoma in a male population. Ophthalmology. 2004; 111(9):1649-1652.

23. Karadimas $P$, Bouzas EA, Topouzis F, Koutras DA, Mastorakos G. Hypothyroidism and glaucoma. A study of 100 hypothyroid patients. Am J Ophthalmol. 2001; 131(1):126-128.
24. Smith KD, Tevaarwerk GJ, Alen LH. Reversal of poorly controlled glaucoma on diagnosis and treatment of hypothyroidism. Can J ophthalmol. 1992; 27(7): 345-347.

25. Smith KD, Tevaarwerk GJ, Alen LH. An ocular dynamic study suporting the hypothesis that hypothyroidism is a treatable cause of secondary open-angle glaucoma. Can J Ophthalmol. 1992; 27(7): 341-344.

26. Bahçeci UA, Ozdek S, Pehlivanli Z, Yetkin I, Onol M. Changes in intraocular pressure and corneal and retinal nerve fiber layer thicknesses in hypothyroidism. Eur $\mathrm{J}$ Ophthalmol. 2005; 15(5):556-561.

27. Rahman I, Cannon PS, Sadiq SA. Tonopen versus Goldmann applanation tonometry for detecting restrictive thyroid eye disease. Ophthal Plast Reconstr Surg. 2010; 26(1):36-38.

28. Martinez-de-la-Casa JM, Garcia-Feijoo J, Fernandez-Vidal A, Mendez-Hernandez C, Garcia-Sanchez J. Ocular response analyzer versus Goldmann applanation tonometry for intraocular pressure measurements. Invest Ophthalmol Vis Sci. 2006; 47(10):4410-4414.

29. Doughty MJ, Zaman ML. Human corneal thickness and its impact on intraocular pressure measures: a review and meta-analysis approach. Surv Ophthalmol. 2000; 44(5):367-408.

30. Konuk O, Onaran Z, Ozhan Oktar S, Yucel C, Unal M. Intraocular pressure and superior ophthalmic vein blood flow velocity in Graves' orbitopathy: relation with the clinical features. Graefes Arch Clin Exp Ophthalmol. 2009; 247(11):1555-1559. 